\title{
Fuego, mortalidad
}

\section{y rebrotación en especies forestales de la}

\section{Sierra Norte de Puebla}

\section{Fire, mortality and resprouting in forest species at Sierra Norte de Puebla}

\author{
Félix Francisco Gómez-Mendoza' y Dante Arturo Rodríguez-Trejo 2*
}

\author{
1 Comisión Nacional Forestal. Centro Regional de \\ Manejo del Fuego. Puebla, Puebla, México. \\ felixforest@hotmail.com
}

\author{
2 Universidad Autónoma Chapingo. División de * Autor de correspondencia. dantearturo@yahoo.com \\ Ciencias Forestales. Chapingo, Edo. de México, \\ México.
}

\section{RESUMEN}

Los incendios forestales están aumentando en el planeta debido al efecto invernadero, por lo que es necesario comprender mejor sus efectos, para lograr un adecuado manejo del fuego. El objetivo de este trabajo fue evaluar y modelar las probabilidades de mortalidad $(\mathrm{Pm})$ y rebrotación $(\mathrm{Pr})$ en Pinus montęumae, Pinus teocote, P. patula, Quercus spp. (Q. crassifolia, Q. rugosa, Q. crassipes), Arbutus xalapensis y Alnus acuminata nueve meses después de un incendio en el ejido Michac, municipio de Chignahuapan, Puebla. Se usaron modelos logísticos y las estimaciones se obtuvieron con el programa SAS. Se logró obtener modelos robustos para la Pm en una especie (dos modelos) y la Pr en tres especies (siete modelos). La Pm fue una función inversa de la altura y el diámetro normal en P. montęumae. Para Pr, se encontró una relación inversa con altura y diámetro normal en $P$. montezumae, Q. spp. y A. xalapensis, así como una relación directa con el porcentaje de copa afectada en $P$. monterumae (todos los modelos con $\mathrm{p} \leq 0.05$ para las variables, límites de confianza sin incluir 1 , concordancia $\geq$ $40 \%$ y p de la prueba de bondad de ajuste $>0.05)$. A pesar de la severidad del fuego, las especies muestran adaptaciones, como la rebrotación, que indican supervivencia. Este tipo de información es de utilidad para estimar mortalidad arbórea por incendios, en quemas prescritas, la elección de sitios para manejo de combustibles y valorar la conveniencia de realizar actividades de reforestación y restauración.

Palabras ClaVe: adaptaciones al fuego, manejo del fuego, Pinus montęumae, regresión logística.

\section{ABSTRACT}

Forest fires are increasing world-wide because of the global warming. So is necessary to understand better its effects to achieve an appropriate fire management. The objective of this work was to evaluate and to model the probabilities of both, mortality (Pm) and resprouting (Pr), on Pinus montezumae, P. teocote, P. patula, Quercus spp. (Q. crassifolia, Q. rugosa and Q. crassipes), Arbutus xalapensis and Alnus acuminata nine months after a forest fire in the ejido Michac, municipality of Chignahuapan, Puebla, Mexico. Were utilized logistic models and the estimations were made with the SAS program. It was possible to obtain robust models of Pm for one species (two models) and Pr models for three species (seven models). The Pm was inversely related to height and diameter to breast height in P. montezumae. For the Pr, there was an inverse relationship with Alt and Dn for P. montezumae, Quercus spp. and Arbutus xalapensis, as well as a direct relationship with percentage of crown fire affected in $P$. montezumae (all models with $\mathrm{p}$ value of the variables in models $\leq 0.05$, confidence limits without including the value 1 , concordance $\geq 40 \%$, and goodness-of-fit test with $p>0.05$ ). Despite the fire severity, the studied species show fire traits, as resprouting ability, that demonstrate its survival. This kind of information is useful to estimate fire-related tree mortality, for prescribed burns, to choose sites for fuel management, and to decide the convenience of conducting reforestation and restoration works.

KEYWORDS: fire traits, fire management, Pinus montęumae, logistic regression. 


\section{INTRODUCCIÓN}

Los incendios forestales han incrementado en extensión, intensidad, severidad y periodo en el que se presentan; este fenómeno se observa en muchas partes del planeta, debido al cambio climático global (World Wide Fund for Nature [WWF] y Boston Consulting Group [BCG], 2020). El fuego ha sido un factor ecológico desde hace más de 400 millones de años cuando comenzaron a presentarse los incendios en la vegetación del planeta (Scott, Bowman, Bond, Pyne y Alexander, 2014). México no es la excepción y aproximadamente $40 \%$ de sus tipos de vegetación y $40 \%$ de la superficie con vegetación en el país, son considerados mantenidos por el fuego, y hay evidencia de incendios por lo menos desde hace 200 millones de años en el actual territorio nacional (Rodríguez-Trejo, 2008; 2014). Hardesty, Myers y Fulks (2005) clasifican los ecosistemas del planeta, por su relación con el fuego, en: mantenidos por, sensibles al, influenciados por e independientes del fuego. El fuego ayuda a mantener a los primeros (dominados por especies adaptadas al fuego), cambia dramáticamente a los segundos (predominan especies sensibles), modifica parcialmente a los terceros (pues tienen tanto especies sensibles como adaptadas al fuego) y en los últimos los incendios no ocurren (Hardesty et al., 2005). El manejo integral y moderno del fuego involucra la prevención y el combate, su uso ecológico y su adecuado uso comunitario, maximizando los beneficios del fuego y reduciendo al mínimo sus efectos negativos (Rodríguez-Trejo, Rodríguez-Aguilar, Fernández-Sánchez y Pyne 2001; Shliski et al., 2007; Rodríguez-Trejo, 2015).

En México, tres de las últimas cuatro temporadas de incendios forestales (2017 a 2020) han sido severas, con 8896, 6970, 7410 y 5913 incendios, que afectaron 726631 ha, 488162 ha, 633678 ha y 378928 ha, respectivamente. En comparación, los promedios de número de incendios y superficie afectada de 2013 a 2020, fueron 7144 incendios y 394649 ha por año (Comisión Nacional Forestal [Conafor], 2020). En el año que ocurrió el incendio bajo estudio (2009), Puebla ocupó el $5^{\circ}$ lugar nacional en número de incendios, con 512 , y el $12^{\circ}$ lugar en superficie afectada (7403 ha) (Conafor, 2009). La intensidad del fuego se refiere a la cantidad de energía que libera por unidad de superficie o por unidad lineal del frente de fuego, mientras que la severidad tiene que ver con los daños que origina, como son mortalidad y efectos en copas (Scott et al., 2014). Los incendios forestales en general pueden mostrar diferentes niveles de afectación, por ejemplo, un incendio severo tendrá áreas afectadas de alta, moderada y baja severidad, aunque prevalezcan las primeras (Agee, 1993), de ahí que se puedan estudiar en un mismo incendio diferentes niveles de afectación.

Las especies forestales pueden considerarse adaptadas o no adaptadas al fuego. En el primer caso, cuentan con mecanismos morfofisiológicos que ayudan a que la especie resista el paso del fuego (como la corteza gruesa de los pinos o la buena poda natural de muchos de ellos) o que lo tolere. En este último caso, el fuego puede consumir la parte aérea, pero yemas que sobreviven en raíces, base del tronco, tronco o copa, permiten la recuperación del individuo por rebrotación, principalmente en latifoliadas (Whelan, 1997; Miller, 2000; New, 2014).

El modelo logístico permite modelar la probabilidad de variables binomiales, por ejemplo, la mortalidad de organismos, como función de variables continuas, discretas o binomiales (Hosmer, Lemeshow y Sturdivant, 2013). Estados Unidos es precursor en la utilización de estos modelos con variables dasométricas y de severidad del fuego como variables explicativas. Una síntesis de esta clase de trabajos en dicho país, donde se han enfocado primordialmente a coníferas, es la de Wooley, Shaw, Ganio y Fitzgerald (2011), quienes señalan que al 2011, se habían desarrollado más de 100 modelos logísticos para estimar probabilidad de mortalidad ( $\mathrm{Pm}$ ) postfuego en 19 especies del oeste de Estados Unidos y que tal herramienta estadística es la más utilizada con ese propósito. Trabajos pioneros son los de Ryan (1982a; 1982b) y de Ryan y Reinhardt (1988), para siete especies de coníferas, entre otros.

México es considerado centro de diversidad de pinos, con aproximadamente $50 \%$ de las especies conocidas en el mundo (Challenger y Soberón, 2008) y, en general, los 
pinos mexicanos, como P. monterumae Lamb. y P. teocote Schiede ex Schltdl. \& Cham., cuentan con adaptaciones al fuego: corteza gruesa, buena poda natural, capacidad de rebrotación en la base, recuperación de la copa afectada, regeneración en áreas incendiadas, así como estado cespitoso en P. montezumae (Rodríguez-Trejo y Fulé, 2003; Rodríguez-Trejo, 2014).

En México se han estudiado diez especies para estimar la $\mathrm{Pm}$ o su probabilidad de rebrotación $(\mathrm{Pr})$ en áreas incendiadas, vía regresión logística: $P$. hartwegii Lindl. (probabilidad de mortalidad, Rodríguez-Trejo, Castro-Solís, Zepeda-Bautista y Carr, 2007; Vera-Vilchis y RodríguezTrejo, 2007; Robles, Velázquez, Rodríguez-Trejo, Reyes y Etchevers, 2016; Hernández-Correa, Rodríguez-Trejo y Cruz-Reyes, 2019), P. montezumae (en este caso relacionado con afectación por fuego y plagas, Fonseca, de los Santos, Llanderal, Cibrián, Rodríguez-Trejo y Vargas, 2008), P. teocote (rebrotación, Juárez, Rodríguez-Trejo y Myers, 2012) P. oocarpa Schiede ex Schltdl. (mortalidad y rebrotación, Pantoja, Rodríguez-Trejo, Myers, Hernández y González, 2018; Rodríguez-Trejo, Martínez-Muñoz y Martínez-Lara, 2019), Abies religiosa (Kunth) Schltdl. \& Cham (mortalidad, Temiño, Rodríguez-Trejo, Molina y Ryan, 2016), Juniperus deppeana Steud. (Rodríguez-Trejo, Pausas y Miranda, 2019), Q. crassifolia Bonpl. (mortalidad y rebrotación, Juárez et al., 2012), Q. magnoliifolia Née (rebrotación, López, RodríguezTrejo, Santiago-Cortés, F., Sereno-Chávez y GranadosSánchez, 2015), Alnus arguta Benth. (rebrotación, DíazHernández, Rodríguez-Laguna, Rodríguez-Trejo, Acevedo-Sandoval y Maycotte-Morales, 2014) y Arbutus xalapensis Kunth (rebrotación, Juárez et al., 2012; DíazHernández et al., 2014).

Es importante evaluar y modelar la mortalidad arbórea postfuego de una especie en diferentes localidades y condiciones ambientales y de la masa, así como de diferentes especies. Además de contar con una cifra de mortalidad postfuego, es conveniente conocer qué características del arbolado, así como de intensidad y severidad del fuego facilitan la mortalidad. Esta información también es de utilidad para realizar quemas prescritas, pues permite conocer las características del arbolado y el nivel de severidad, por ejemplo, indicado por la cicatriz de quemado sobre el tronco o el porcentaje de afectación a la copa que no deben rebasarse para evitar mortalidad o que esta sea muy baja al aplicar una quema prescrita.

\section{OBJETIVOS}

El objetivo del presente trabajo fue evaluar y modelar las probabilidades de mortalidad y de rebrotación de individuos de $P$. montezumae, $P$. teocote, $P$. patula Schiede ex Schltdl. \& Cham, Quercus spp. (Q. crassifolia, Q. rugosa, Q. crassipes), Arbutus xalapensis Kunth y Alnus acumuniata Kunth, nueve meses después de un incendio forestal en la Sierra Norte de Puebla, México.

\section{MATERIALES Y MÉTODOS}

\section{Área de estudio}

El estudio se llevó a cabo en un área afectada por incendio forestal, en el NO del ejido Michac, del Municipio de Chignahuapan, en la Sierra Norte de Puebla, México. Las coordenadas del área de estudio son: $19^{\circ} 52^{\prime} 8.7^{\prime \prime}$ a $19^{\circ} 52^{\prime}$ 49.4” N y $98^{\circ} 05^{\prime} 26.3^{\prime \prime}$ a $98^{\circ} 06^{\prime} 04.2^{\prime \prime}$ O. El clima, de acuerdo con la clasificación de Köppen modificada por García de Miranda (1998) corresponde a templado subhúmedo con lluvias en verano $\mathrm{Cbm}(\mathrm{f})(\mathrm{e}) \mathrm{gw}$ ", temperatura media anual de $14.6{ }^{\circ} \mathrm{C}$ y una precipitación media anual de $1067 \mathrm{~mm}$. La topografía es accidentada, montañosa, los suelos prevalecientes son andosoles y la vegetación dominante el bosque de pino (Centro Nacional de Estudios Municipales [CNEM], 1987).

El incendio inició el 26 de marzo de 2009 y fue controlado el 28 de marzo de 2009. Cubrió una superficie de 350 ha, de las cuales 180 ha incluyeron renuevo y arbolado en diferentes etapas de desarrollo (Conafor, 2009). Se enclavó entre las cotas altitudinales $2280 \mathrm{~m}$ a $2650 \mathrm{~m}$ s.n.m., principalmente sobre terrenos con pendiente moderada $(<30 \%)$. 


\section{Muestreo}

Se desarrolló un muestreo sistemático durante el periodo del 5 de diciembre al 3 de enero de 2010, nueve meses después del incendio. Se levantaron un total de 83 sitios circulares de $1000 \mathrm{~m}^{2}$, un sitio cada $100 \mathrm{~m}$, delimitados con cuerda compensada, para una superficie de muestra igual a 8.3 ha, que representa $2.3 \%$ de intensidad de muestreo. Se obtuvo una muestra de 1863 árboles $>1 \mathrm{~m}$ de altura de varias especies, de los cuales 216 corresponden a $P$. montezumae, 1273 P. teocote, 73 P. patula, 204 A. xalapensis, 42 A. acuminata y 55 Quercus spp. En el último caso, se hallaron tres especies (Q. crassifolia Benth., Q. rugosa Née y Q. crassipes Bonpl.) y dado el escaso número de individuos, se agruparon como género. No se logró modelar a $P$. patula (por su total mortalidad) ni a $A$. acuminata (por sus casi totales supervivencia y rebrotación).

Los datos tomados por árbol fueron: especie, si estaba vivo o muerto (copa totalmente quemada o deshidratada por el fuego y sin seña de rebrotes de hojas ni rebrotes en la copa o en la base del tronco), altura total (Alt, m) (medida con baliza o clinómetro), diámetro normal (Dn, cm) (obtenido con cinta diamétrica o forcípula), altura original a la base de la copa (Abc, m) (medida con baliza o clinómetro) y número de rebrotes en la base del tronco. Asimismo, las variables de severidad del fuego registradas por árbol fueron: altura de la cicatriz del fuego sobre el tronco (m) y el porcentaje de afectación del volumen de la copa (Pac, quema o deshidratación) estimada de forma visual. En cada sitio se registraron paraje, coordenadas y altitud con un GPS (Garmin III Plus), pendiente con un clinómetro y exposición con una brújula.

\section{Análisis y modelo estadístico}

La Pm y la Pr en cada especie fueron estimadas mediante regresión logística utilizando como variables independientes a Alt, Dn, Abc y a las variables de severidad. El modelo logístico empleado fue el de Hosmer et al. (2013) (Ecuación 1):

$$
\mathrm{P}=1 /\left(\left(1+\exp -\left(\alpha+\beta_{1} \mathrm{~V}_{1}+\beta_{2} \mathrm{~V}_{2}+\ldots+\beta_{\mathrm{n}} \mathrm{V}_{\mathrm{n}}\right)\right)\right.
$$

donde:

$\mathrm{P}=$ probabilidad de mortalidad o de rebrotación

$\alpha=$ intercepto

$\beta_{1}=$ constante asociada a la variable 1

$\mathrm{V}_{1}=$ variable 1

$\beta_{2}=$ constante asociada a la variable 2

$\mathrm{V}_{2}=$ variable 2

$\beta_{\mathrm{n}}=$ constante asociada a la variable $\mathrm{n}$

$\mathrm{V}_{\mathrm{n}}=$ variable $\mathrm{n} ; \mathrm{n}$ representa el número de variables incluidas en el modelo

El ajuste de los modelos se realizó con el procedimiento logístico (PROC LOGISTIC) del programa SAS (Statistical Analysis System) para microcomputadoras, v. 9.4 (SAS Institute, 2012). Los modelos fueron probados con cada una de las variables dasométricas y de severidad: individualmente, en combinaciones de dos en dos, de tres en tres y en su totalidad. Para que un modelo se considerase significativo, todas sus variables tuvieron que ser significativas ( $\mathrm{p} \leq 0.05)$, su límite de confianza sin incluir la unidad (lo que implicaría una razón de momios probabilidad de ocurrencia entre la de no ocurrencia- igual a 1), y concordancia relevante, de $40 \%$ o más. Finalmente, se probó la bondad de ajuste de los modelos con la prueba de Hosmer-Lemeshow, también en SAS, que cuando resulta no significativa $(\mathrm{p}>0.05)$ indica que las probabilidades pronosticadas no se desvían de las probabilidades observadas de una manera que la distribución binomial no predice.

\section{Estimación de la intensidad del incendio}

La intensidad del fuego se estimó, de manera aproximada, bajo el supuesto de que la altura de la cicatriz del fuego sobre el tronco es equivalente a la longitud de llama. Se usó el modelo de Byram (1959) (Ecuación 2) y se despejó para obtener la intensidad calorífica (Ecuación 3). Se tomaron en cuenta solo los árboles que presentaron dicha cicatriz.

$$
\mathrm{L}=0.0775(\mathrm{IB}) 0.46
$$

$$
\mathrm{IB}=\operatorname{alog}((\log \mathrm{L}-\log 0.0775) / 0.46)
$$


donde:

$\mathrm{L}=$ longitud de llama $(\mathrm{m})$

$\mathrm{IB}=$ intensidad de Byram $\left(\mathrm{kW} \mathrm{m}^{-1}\right)$

Estos componentes del comportamiento del fuego solo fueron estimados donde había $P$. teocote y $P$. montezumae, las especies más abundantes (79.9\% entre ambas) y en los cuales se pudo distinguir con mayor claridad la cicatriz de quemado sobre el tronco.

\section{Resultados}

\section{Características de la masa y severidad del fuego}

La distribución de la muestra incluyó árboles desde juveniles hasta maduros en las especies estudiadas, principalmente pinos (Tabla 1). El menor porte de las latifoliadas se explica en parte porque se trata de áreas bajo manejo forestal, donde tienden a ser controladas silvícolamente para favorecer a los pinos, que son de mayor interés comercial. A partir del modelo de Byram (1959) y despejándolo, se estimó la intensidad lineal del fuego para P. teocote:

$$
\mathrm{IB}=\operatorname{alog}((\log 2.82-\log 0.0775) / 0.46)=2473.8 \mathrm{~kW} \mathrm{~m}^{-1}
$$

El promedio de la altura de la cicatriz del fuego sobre el tronco en P. montezumae $(2.57 \mathrm{~m})$, fue ligeramente menor y la intensidad equivalente a $2021.72 \mathrm{~kW} \mathrm{~m}^{-1}$.

La mortalidad resultó heterogénea entre las especies estudiadas. En P. montezumae fue $26.4 \%$, pero en $P$. teocote alcanzó $61.5 \%$ y $100 \%$ en P. patula. Entre las especies latifoliadas la mortalidad fue más reducida: 9.3\% para $A$. xalapensis, 2.4\% en $A$. acuminata y 5.5\% en Quercus spp.

TABLA 1. Variables dasométricas y de severidad del fuego en las especies estudiadas.

\begin{tabular}{|c|c|c|c|c|c|c|c|c|c|c|c|c|c|c|c|}
\hline \multirow{2}{*}{ Variable } & \multicolumn{3}{|c|}{ P. montezumae } & \multicolumn{3}{|c|}{ P. teocote } & \multicolumn{3}{|c|}{ Arbutus xalapensis } & \multicolumn{3}{|c|}{ Alnus acuminata } & \multicolumn{3}{|c|}{ Quercus spp. } \\
\hline & $\begin{array}{c}\text { Media } \pm \\
D E\end{array}$ & $M$ & $m$ & $\begin{array}{c}\text { Media } \\
\pm D E\end{array}$ & $M$ & $m$ & $\begin{array}{c}\text { Media } \\
\pm D E\end{array}$ & $M$ & $m$ & $\begin{array}{c}\text { Media } \\
\pm D E\end{array}$ & $M$ & $m$ & $\begin{array}{c}\text { Media } \\
\pm D E\end{array}$ & $M$ & $m$ \\
\hline Alt (m) & $\begin{array}{c}9.46 \pm \\
11.5\end{array}$ & 50 & 0.1 & $\begin{array}{c}4.68 \pm \\
8.6\end{array}$ & 50 & 0.3 & $\begin{array}{c}2.57 \pm \\
2.0\end{array}$ & 20 & $\begin{array}{l}0 . \\
4\end{array}$ & $\begin{array}{c}3.85 \pm \\
2.2\end{array}$ & $\begin{array}{l}7 . \\
0\end{array}$ & 1.0 & $\begin{array}{c}2.86 \pm \\
3.4\end{array}$ & 15 & 0.15 \\
\hline $\operatorname{Dn}(\mathrm{cm})$ & $\begin{array}{c}14.82 \pm \\
16.4\end{array}$ & 60 & 1.0 & $\begin{array}{c}7.43 \pm \\
11.8\end{array}$ & 60 & 1.0 & $\begin{array}{c}4.70 \pm \\
3.7\end{array}$ & 40 & 1 & $\begin{array}{c}6.48 \pm \\
5.0\end{array}$ & $\begin{array}{l}2 \\
5 . \\
0\end{array}$ & $\begin{array}{l}2 . \\
0\end{array}$ & $\begin{array}{c}6.58 \pm \\
8.9\end{array}$ & 25 & 1.0 \\
\hline$A b c(m)$ & $\begin{array}{c}0.38 \pm \\
1.8\end{array}$ & 20 & 0.5 & $\begin{array}{l}0.1 \pm \\
0.5\end{array}$ & 7 & 0.5 & $\begin{array}{c}0.01 \pm \\
0.1\end{array}$ & 1 & 0 & $\begin{array}{c}0.15 \pm \\
0.5\end{array}$ & 1.5 & 1.5 & $\begin{array}{c}0.24 \pm \\
1.4\end{array}$ & 10 & 0 \\
\hline Acft (m) & $\begin{array}{c}0.96 \pm \\
1.5\end{array}$ & 7 & 0 & $\begin{array}{c}0.35 \pm \\
1.0\end{array}$ & 8 & $\mathrm{O}$ & $\begin{array}{c}0.05 \pm \\
0.4\end{array}$ & 6 & 0 & & & & $\begin{array}{c}0.16 \pm \\
0.9\end{array}$ & 7 & 0 \\
\hline $\operatorname{AcftP}(\mathrm{m})$ & $\begin{array}{c}2.57 \pm \\
1.4\end{array}$ & 7 & 0 & $\begin{array}{c}2.82 \pm \\
1.3\end{array}$ & 8 & 0.5 & $\begin{array}{c}0.05 \pm \\
0.4\end{array}$ & 6 & 0 & & & & $\begin{array}{c}4.5 \pm \\
3.5\end{array}$ & 7 & 2.0 \\
\hline $\operatorname{Pac}(\%)$ & $\begin{array}{c}34.35 \pm \\
45.6\end{array}$ & 100 & 0 & $\begin{array}{c}29.4 \pm \\
44.3\end{array}$ & 100 & $\mathrm{O}$ & $\begin{array}{c}87.0 \pm \\
0.4\end{array}$ & 100 & 0 & $\begin{array}{c}96.7 \pm \\
15.4\end{array}$ & 0 & 0 & $\begin{array}{c}73.8 \pm \\
43.6\end{array}$ & $\begin{array}{c}10 \\
0\end{array}$ & 0 \\
\hline
\end{tabular}

Alt = altura, Dn = diámetro normal, Abc = altura a la base de la copa, Acft = altura de la cicatriz del fuego sobre el tronco, AcftP = altura de la cicatriz del fuego sobre el tronco donde se presentó, Pac = porcentaje de afectación de la copa. DE = desviación estándar, M = máximo, m = mínimo.

Nota: como P. patula tuvo $100 \%$ de mortalidad, sólo se le registró Alt $(4.51 \mathrm{~m} \pm 1.77 \mathrm{~m})$ y $\mathrm{Dn}(4.04 \mathrm{~cm} \pm 1.37 \mathrm{~cm})$. 


\section{Probabilidad de mortalidad, Pinus montezumae}

Los modelos que resultaron significativos para Pm $(\mathrm{p} \leq$ 0.05 , límites de confianza sin la unidad, concordancia $\geq$ $40 \%$ y prueba de bondad de ajuste con $\mathrm{p}>0.05)$, fueron el 5 y el 6 (Ecuaciones 5 y 6 ) (la simbología se proporciona al pie de la tabla 1):

$$
\begin{gathered}
\operatorname{Pm}=1 /((1+\exp -(0.2469-0.3919 \mathrm{Alt})) \\
\mathrm{Pm}=1 /((1+\exp -(0.578-0.2589 \mathrm{Dn}))
\end{gathered}
$$

La figura 1 muestra escenas de la afectación al arbolado. La significancia de los parámetros es presentada en la tabla 2 y la probabilidad de mortalidad en función a Alt y Dn se observa en la figura 2 .

\section{Probabilidad de rebrotación, P. montezumae}

La rebrotación media obtenida fue de $25.5 \%$. La altura, el diámetro normal y la proporción de copa afectada al fuego, generaron modelos significativos de probabilidad de rebrotación (Pr) (Ecuaciones 7 a 9):

$$
\begin{aligned}
& \operatorname{Pr}=1 /((1+\exp -(0.2954-0.4457 \text { Alt })) \\
& \operatorname{Pr}=1 /((1+\exp -(0.5904-0.2715 \mathrm{Dn})) \\
& \operatorname{Pr}=1 /((1+\exp -(-3.2376+0.0289 \mathrm{Pac}))
\end{aligned}
$$

La significancia del modelo y parámetros, se muestran en la tabla 3, y los gráficos derivados de los modelos, en la figura 3.

\section{Probabilidad de rebrotación, A. xalapensis}

En este caso, rebrotaron $89 \%$ de los árboles y fueron significativos dos modelos (10 y 11):

$$
\begin{aligned}
& \operatorname{Pr}=1 /((1+\exp -(2.5619-0.1752 \mathrm{Alt})) \\
& \operatorname{Pr}=1 /((1+\exp -(2.8401-0.1461 \mathrm{Dn}))
\end{aligned}
$$

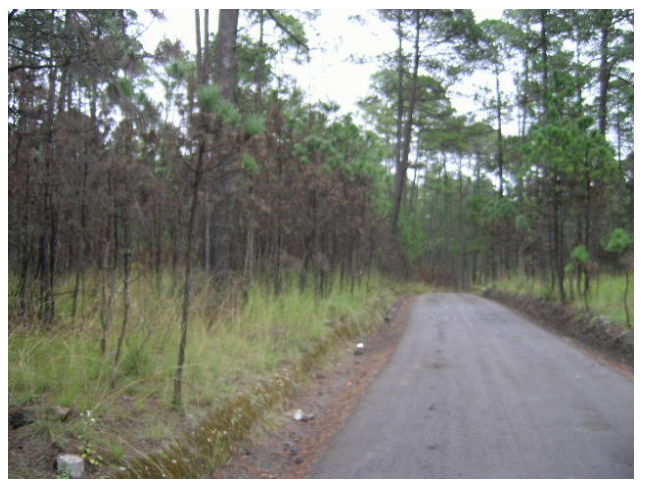

A

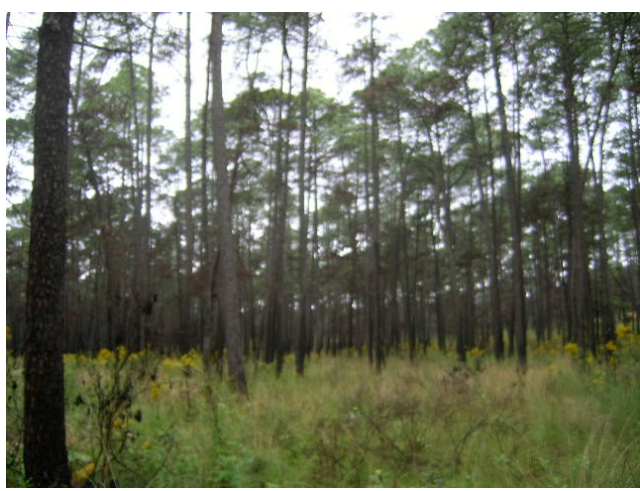

B

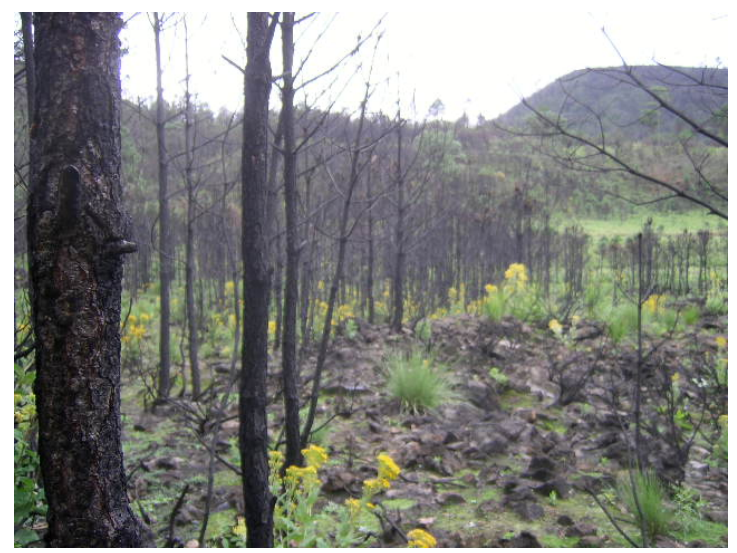

FiguRA 1. A) Pinar (P. montezumae) maduro afectado a severidad moderada. B) Pinar joven afectado a mediana severidad. C) Pinar ( $P$. patula) de pequeñas dimensiones y denso, donde el fuego tuvo alta severidad (mortalidad). 
TABLA 2. Significancia de los modelos de probabilidad de mortalidad para P. montezumae (modelos 5 y 6 ).

\begin{tabular}{cccccc}
\hline Modelo & Pintercepto & Pvariable(s) & IC & C (\%) & PBAHL \\
\hline 5 & 0.3274 & 0.0005 & $0.542-0.483$ & 74.0 & 0.1278 \\
6 & 0.0534 & $<0.0001$ & $0.68-0.876$ & 76.7 & 0.2377 \\
\hline
\end{tabular}

IC = intervalo de confianza a 95\%, C = concordancia, p BAHL = valor de $\mathrm{p}$ de la prueba de bondad de ajuste de Hosmer y Lemeshow.

A

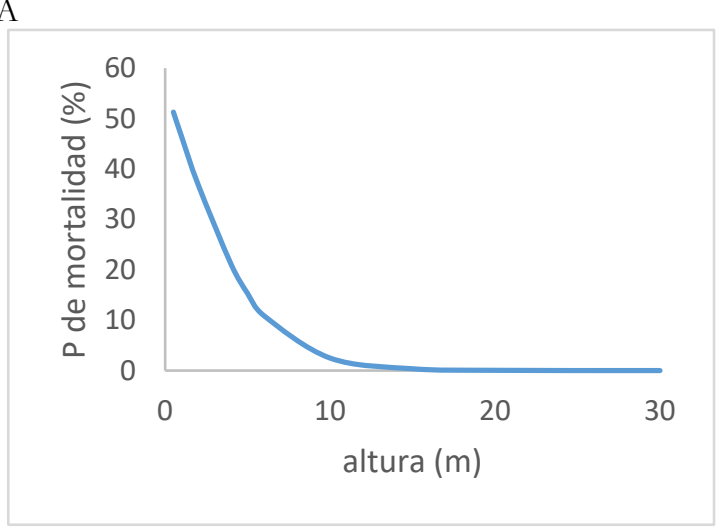

B

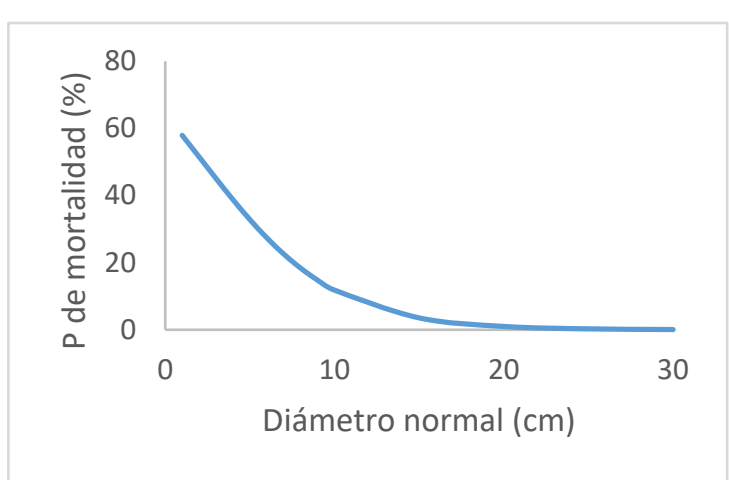

Figura 2. A) Probabilidad de mortalidad postincendio para P. montezumae, como función de su altura (Ecuación 5) y B) como función de su diámetro normal (Ecuación 6).

TABLA 3. significancia de los modelos de probabilidad de rebrotación para P. montezumae (modelos 7 a 9), A. xalapensis (10 y 11) y Quercus spp. (12 y 13).

\begin{tabular}{ccccccc}
\hline Modelo & Pintercepto & P variable(s) & & IC & C (\%) & P BAHL \\
\hline 7 & 0.2564 & 0.0004 & 0.501 & -0.819 & 80.0 & 0.2780 \\
8 & 0.0531 & $<0.0001$ & 0.667 & -0.881 & 79.9 & 0.8570 \\
9 & $<0.0001$ & $<0.0001$ & 1.017 & -1.041 & 57.0 & 0.2089 \\
10 & $<0.0001$ & 0.0334 & 0.714 & -0.986 & 56.4 & 0.2081 \\
11 & $<0.0001$ & 0.0102 & 0.772 & -0.966 & 52.6 & 0.9210 \\
12 & $<0.0001$ & 0.0024 & 0.369 & -0.807 & 78.0 & 0.3292 \\
13 & $<0.0001$ & 0.0008 & 0.683 & -0.984 & 68.3 & 0.4069
\end{tabular}


Gómez-Mendoza y Rodríguez-Trejo. Fuego, mortalidad y rebrotación en especies forestales

A

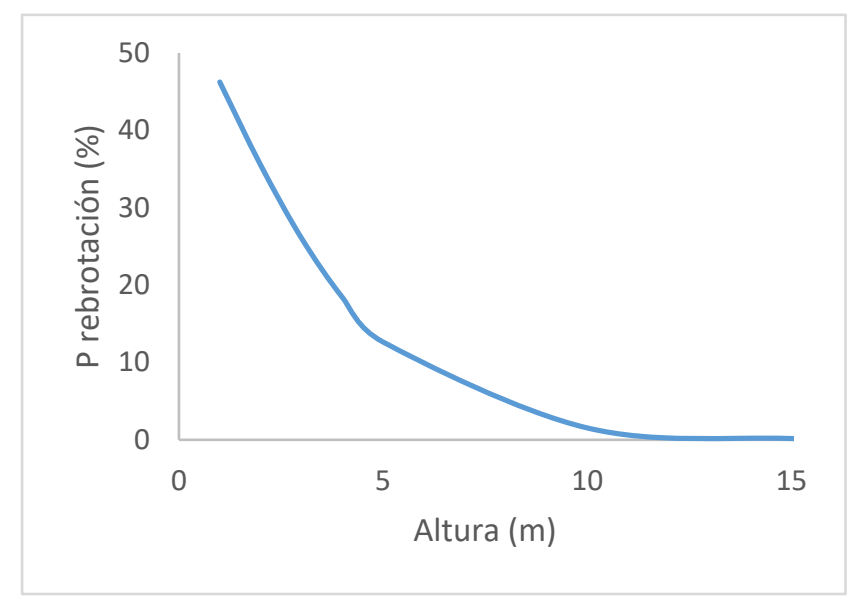

B

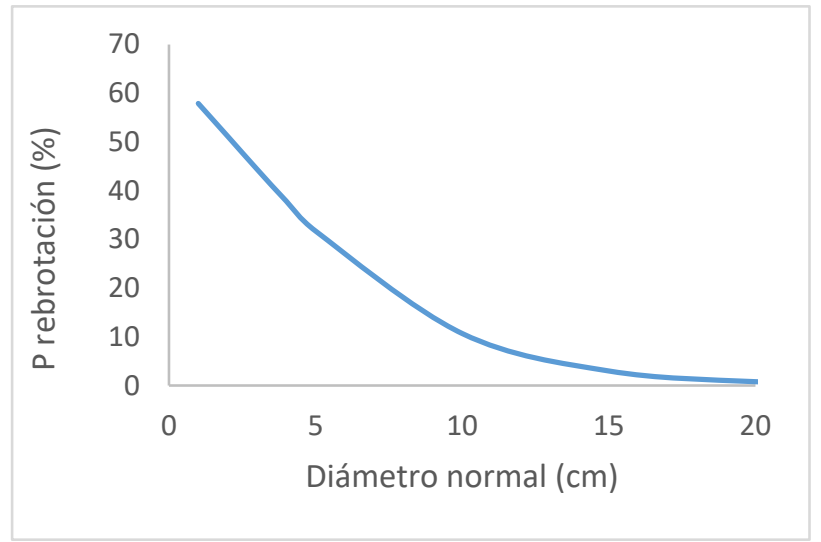

C

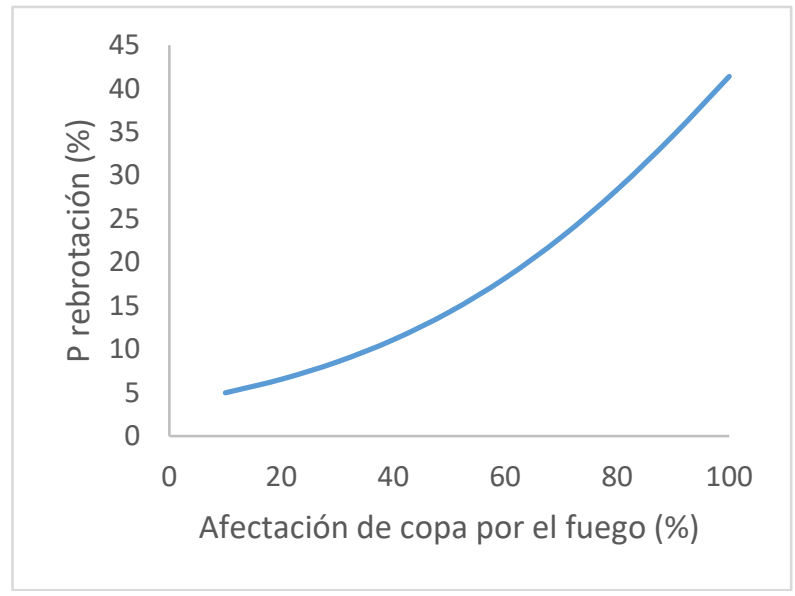

Figura 3. Probabilidad de rebrotación para $P$. montezumae, como función de: A) la altura (modelo 7), B) diámetro normal (Ecuación 8) y C) la proporción de copa afectada al fuego (Ecuación 9). 
Las significancias se muestran en la tabla 3 y los gráficos se exhiben en la figura 4.

\section{Probabilidad de rebrotación, Quercus spp.}

De los encinos rebrotó $76 \%$. Los modelos significativos fueron (12 y 13):

$$
\begin{aligned}
& \operatorname{Pr}=1 /((1+\exp -(3.0624-0.6059 \mathrm{Alt})) \\
& \operatorname{Pr}=1 /((1+\exp -(2.6332-0.2193 \mathrm{Dn}))
\end{aligned}
$$

Las significancias están en la tabla 3 y los gráficos en la figura 5. La figura 6 muestra imágenes de varias especies estudiadas en rebrotación.

\section{DISCUSIÓN}

La intensidad del fuego estimada indica un incendio lo suficientemente intenso para no permitir su combate directo en diversas áreas (com. pers. combatientes, 2012) y, según Agee (1993), ese tipo de combate está limitado a longitudes de llama de hasta $1 \mathrm{~m}(258 \mathrm{~kW} / \mathrm{m})$. El último autor, establece que los incendios de copa superan 2800 $\mathrm{kW} / \mathrm{m}$ y 3 m como longitud de llama.

Pinus montezumae y $P$. teocote son especies adaptadas al fuego. La primera se regenera bien en áreas incendiadas, cuenta con estado cespitoso (amplio desarrollo de follaje que se recupera después del incendio, poco crecimiento en altura pero marcado crecimiento en diámetro y de raíz, que facilitan la recuperación postfuego del brinzal), después del cual crece con relativa rapidez (lo que le permite a la yema apical quedar fuera del alcance de las llamas en incendios que no sean muy intensos) y tiene una buena poda natural, que resta continuidad vertical a los combustibles y reduce la probabilidad de incendio de copas. Asimismo, exhibe capacidad de rebrotación, tanto en la base como de su follaje cuando juvenil o de la copa cuando adulto. Además, presenta una gruesa corteza que protege al cambium de altas temperaturas (Rodríguez-Trejo y Fulé, 2003; Rodríguez-Trejo, 2014).

Pinus teocote es una especie que tiene buena regeneración en áreas incendiadas, rápido crecimiento, corteza gruesa y recupera su copa afectada al fuego. Ambas especies de pinos son resistentes al fuego (Rodríguez-Trejo y Fulé, 2003); en cambio, A. xalapensis, $A$. acuminata y Quercus spp., con rebrotación postfuego más vigorosa y abundante que en los pinos (Juárez et al., 2012; DíazHernández et al., 2014; Rodríguez-Trejo, 2014), se consideran más bien tolerantes al fuego (Whelan, 1997).

Los rebrotes epicórmicos, observados en pinos estudiados, aprovechan que se mantiene la estructura del árbol, representan una importante adaptación al fuego y se han observado en pinos como P. canariensis C. Sm. Ex DC. de las Islas Canarias (Keeley y Pausas, 2017). Se estima que la corteza se desarrolló en los pinos hace unos 126 millones de años, y que se engrosó al mismo tiempo que apareció la serotinidad en los conos, hace 89 millones de años, como respuesta a incendios catastróficos durante el Cretácico (Tinhua, Pausas, Belcher, Schwilk y Lamont, 2012).

La relación inversa que mostró la Pm con la altura y el diámetro en $P$. montezumae es consistente con las tendencias halladas en P. hartwegii (Rodríguez-Trejo et al., 2007; Robles et al., 2016 y Hernández-Correa et al., 2019), P. montezumae afectado por fuego y plagas (Fonseca et al., 2008), P. oocarpa (Rodríguez-Trejo et al., 2019) y Abies religiosa (Temiño et al. 2016). Esta tendencia se relaciona con que, en general, a mayor diámetro de los árboles, el grosor de corteza también aumenta y esta provee mejor aislamiento térmico al cambium (Miller, 2000). Una medida de tal aislamiento, la proporciona Ryan (1990), quien refiere que, bajo condiciones naturales de combustibles forestales en el noroeste de Estados Unidos, las coníferas sobreviven al fuego si tienen por lo menos $1 \mathrm{~cm}$ de grosor de corteza. Aunque en el presente estudio no se halló relación entre indicadores de severidad y mortalidad, en otros sí. Por ejemplo, Rodríguez-Trejo et al. (2019) encontraron relación directa entre altura de la cicatriz sobre el tronco y Pm en J. deppeana de la Reserva de Tehuacán-Cuicatlán, Puebla.

Es posible que, bajo condiciones naturales, $P$. patula tenga regímenes de fuegos de copas, que producen muy alta mortalidad en el arbolado, y que las semillas que se mantengan viables dentro de los conos serótinos recolonicen las áreas afectadas por el fuego, pues la especie 
tiene muy baja capacidad de rebrotación postfuego, tanto en tronco como en copa (Rodríguez-Trejo, 2014), tendencia consistente con lo observado para dicha especie en el presente estudio. Esta estrategia, considerada de semilladores postfuego y no de especies resistentes o tolerantes a este, con cohortes de ciclo de vida monopírico por incendios catastróficos (Pausas, 2015), se observa en especies serótinas como P. contorta Douglas o P. banksiana Lamb. (Miller, 2000).

Con una cicatriz sobre el tronco de $2.57 \mathrm{~m}$ en promedio (máximo igual a $7 \mathrm{~m}$ ), la Pm en P. montezumae es de solo $2.5 \%$ para árboles de $10 \mathrm{~m}$ de altura; así como de $15.3 \%$ para árboles de $5 \mathrm{~m}$ y en árboles con $0.5 \mathrm{~m}$ de altura, que van saliendo del estado cespitoso, tal probabilidad es de 51.3\%.

A

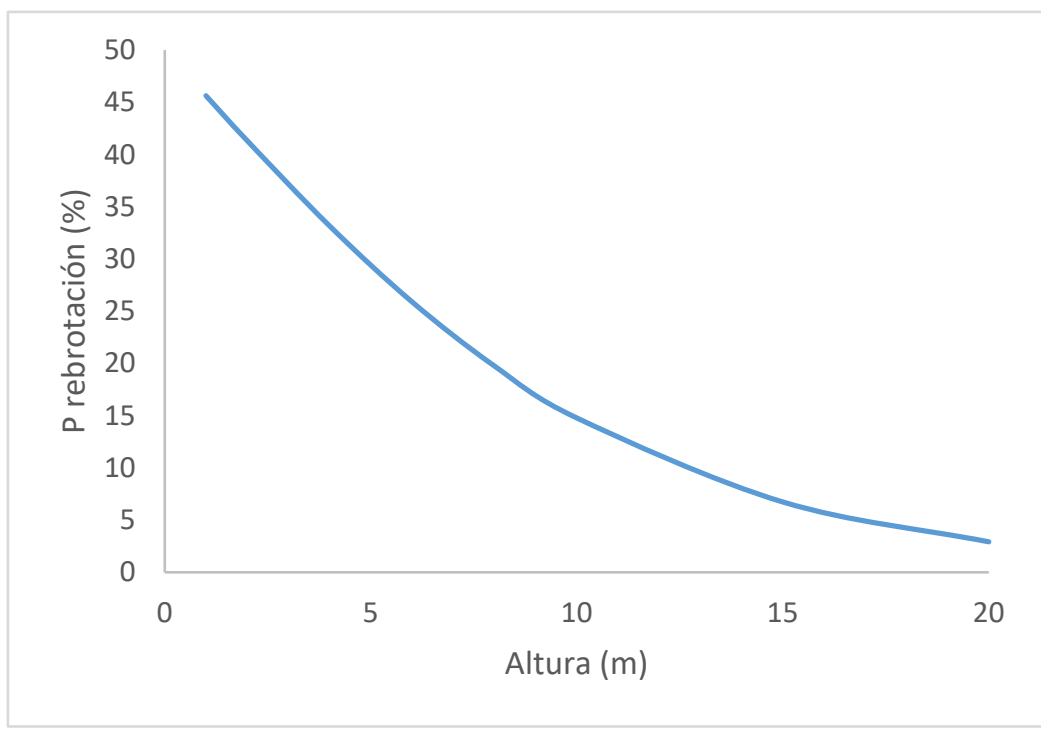

$\mathrm{B}$

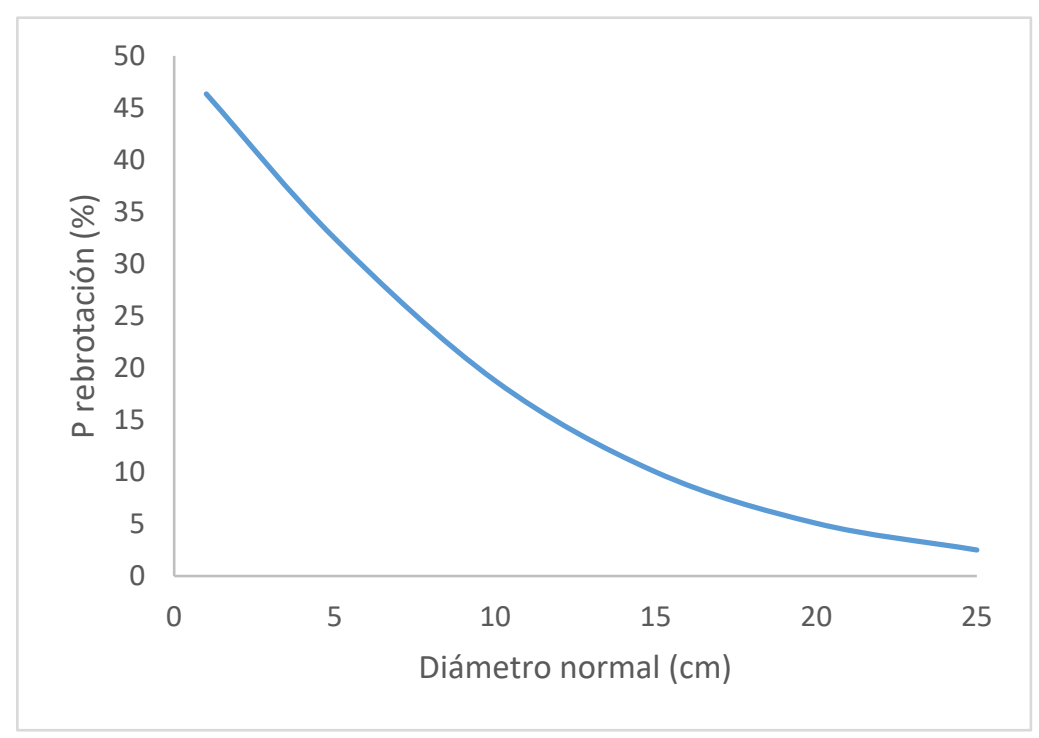

FIgURA 4. Probabilidad de rebrotación para Arbutus xalapensis (ecuaciones 10 y 11), como función de Alt (A) y Dn (B). 
A

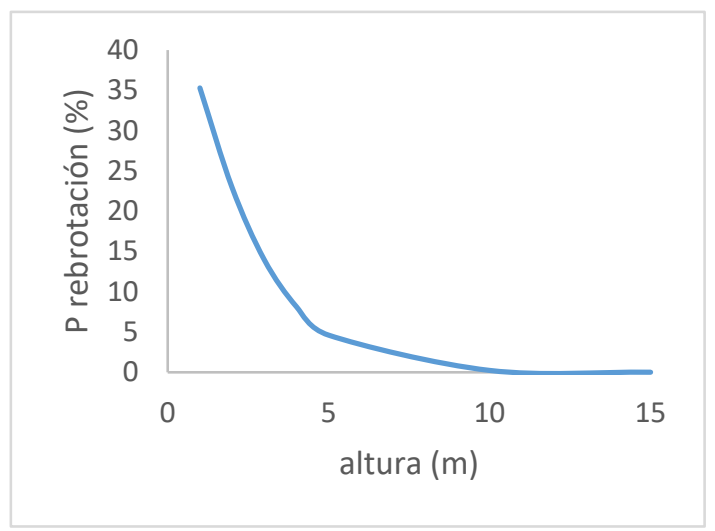

B

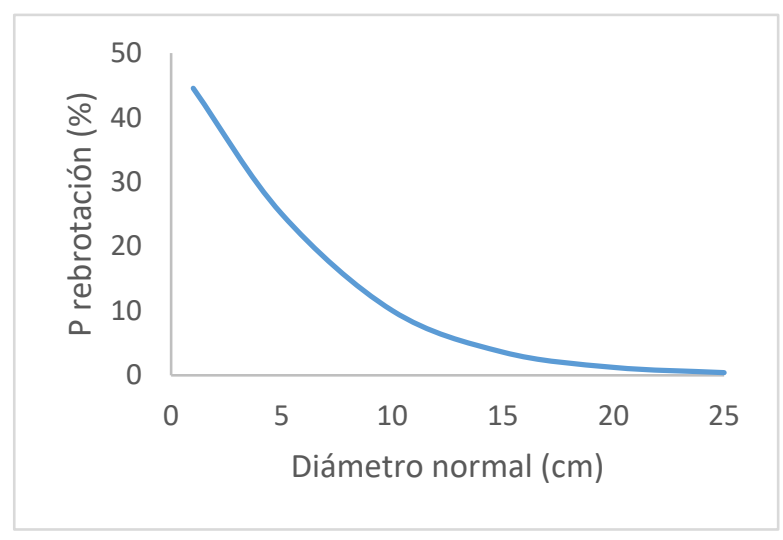

FiguRA 5. A) Probabilidad de rebrotación de Quercus spp., como función de la altura (ecuación 12) y B) como función del diámetro normal (Ecuación 13).

A

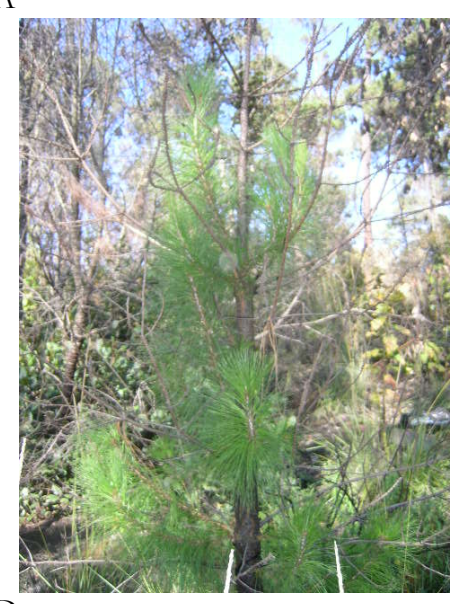

$\mathrm{D}$

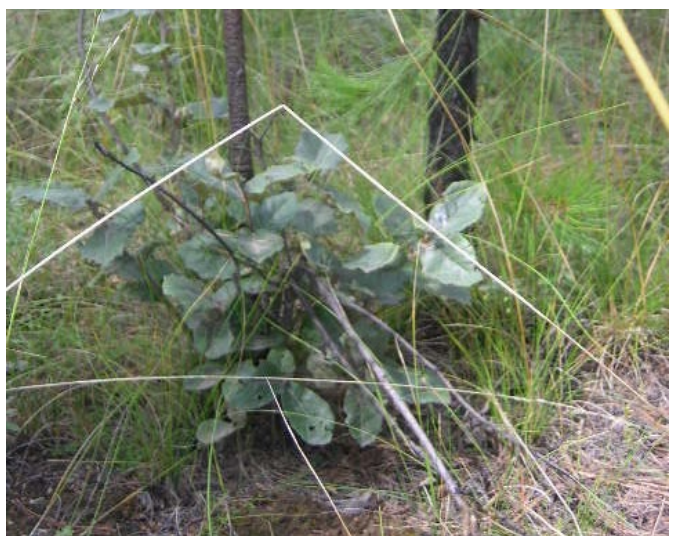

B

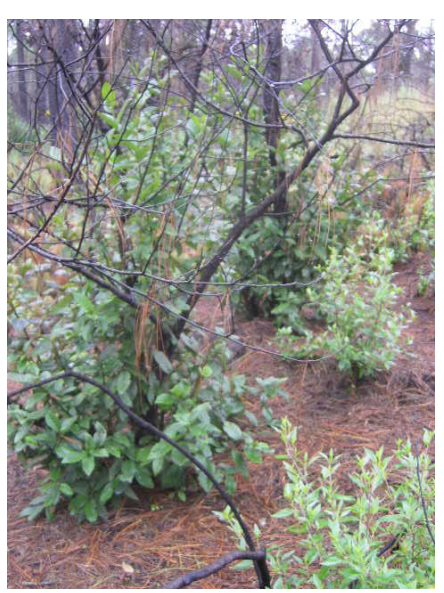

$\mathrm{E}$
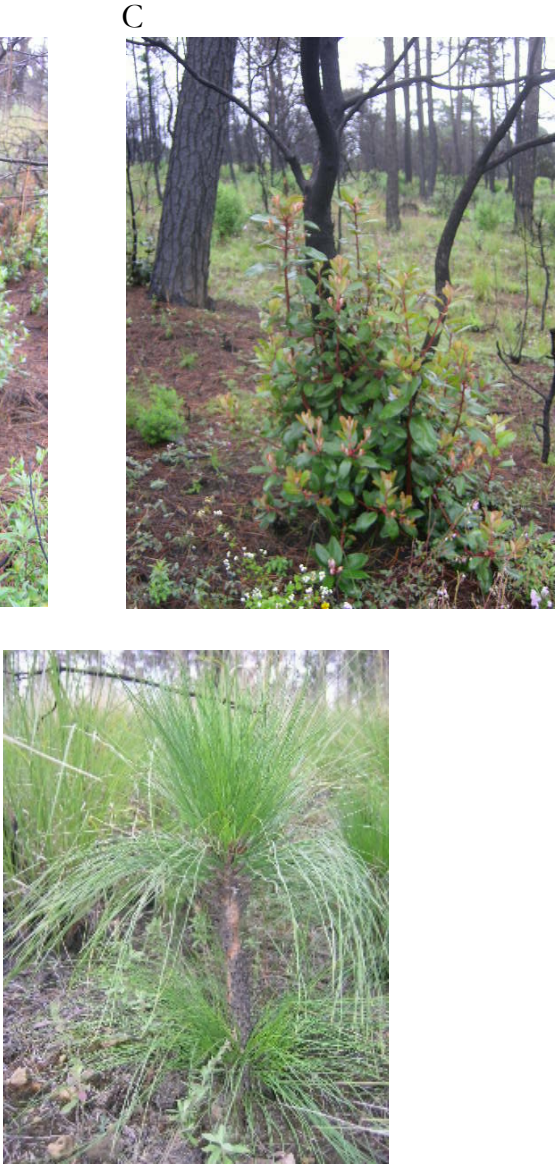

Figura 6. Rebrotación en A) Pinus teocote, B) Alnus acuminata, C) Arbutus xalapensis, y D) Quercus crassifolia. E) Individuo joven de $P$. montezumae recuperado y con rebrotes en la base. 
Asimismo, la Pr mostró patrones similares a los hallados en otras especies. En las tres especies para las que se obtuvieron estos modelos se halló relación inversa entre tal probabilidad y la altura y el diámetro. Es decir, los individuos más jóvenes tienen mayor poder de rebrotación que los viejos. Esta característica es denominada “juvenilidad” (Hartmann, Kester, Davies y Geneve, 2011), se toma en cuenta para realizar propagación vegetativa. Simplemente los tejidos más jóvenes son más vigorosos, más resilientes, ante daños causados por perturbaciones.

Pero la Pr también mostró relación directa con el porcentaje de la copa afectado al fuego en P. montezumae. Mientras más afectada la copa, mayor la rebrotación. Esta respuesta también es común, pues tanto en la base del tronco como sobre este, hay yemas dormantes, protegidas por la corteza, las cuales se activan después del paso del fuego (Miller, 2000; Juárez et al., 2012; Scott et al., 2014). Esta respuesta se debe a la dominancia apical, que controla el crecimiento de las yemas apicales. En ella, hormonas que controlan el crecimiento, en especial auxinas, son producidas en las puntas de los troncos y ramas y hojas adyacentes, hormonas que son traslocadas a las yemas dormantes, lo que previene su activación. Si dichas puntas (o parte de ellas) son eliminadas, por el fuego en este caso, se remueve la fuente de inhibición. La raíz envía citocininas a las yemas dormantes, cuyo balance hormonal cambia luego del incendio y esto permite su activación, dando lugar a la rebrotación (Miller, 2000).

En México se tienen registrados formalmente hasta ahora 42 encinos, arbóreos o arbustivos, con rebrotación postfuego, entre ellos las tres especies aquí estudiadas, si bien se estima que la gran mayoría de las especies nacionales presentan tal respuesta, su rebrotación es principalmente en yemas del cuello de la raíz (Rodríguez-Trejo, 2014). En EE. UU. las especies del género Alnus rebrotan de las yemas adventicias, de las yemas en el cuello de la raíz y de la raíz, en ese orden, ante incendios que van de menor a mayor severidad; por ejemplo, Alnus incana (L.) Moench (Fryer, 2011) o A. rhombifolia Nutt. (Fryer, 2014). Los A. acuminata y $\operatorname{los} A$. xalapensis del presente estudio, sufrieron una severidad regular, pues se perdió su parte aérea, pero se comenzó a generar una nueva vía rebrotación de las yemas en el cuello de la raíz. No se llegó al extremo de severidad que pudiera inducir la rebrotación desde las raíces o la muerte de estos árboles, pues Díaz-Hernández et al. (2014) señalan alta mortalidad de $A$. arguta, en Hidalgo, en zonas afectadas con alta severidad.

Alnus acuminata es una especie secundaria, pionera en bosques de pino, encino o pino-encino de la región central del país (Comisión Nacional para el Conocimiento y Uso de la Biodiversidad [Conabio], 2021). Q. crassifolia se halla en bosques perturbados, mientras que Q. crassipes y Q. rugosa se encuentran tanto en sitios perturbados como no perturbados (Zavala, 2007; Romero, Rojas y Rubio, 2015). El que estas especies se hallen en sitios alterados indica que colonizan tales áreas o bien que sobreviven al fuego y permanecen en la localidad. Como en el presente estudio, Juárez et al. (2012) hallaron que Q. crassifolia tiene una relación inversamente proporcional entre la probabilidad de rebrotación y la altura y el diámetro de los árboles. Asimismo, en los árboles supervivientes al fuego, a mayor afectación del tronco, mayor la probabilidad de rebrotación. Dichos autores también encontraron alta probabilidad de mortalidad de la parte aérea de los encinos, pero baja en los árboles completos, pues se recuperaron mediante rebrotación.

En el presente estudio se obtuvo un modelo en el cual la densidad de los árboles estuvo inversamente relacionada con la probabilidad de mortalidad para todas las especies en conjunto. Sin embargo, dicho modelo no pasó la prueba de bondad de ajuste y por ello no se incluyó aquí. No obstante, en otros estudios del país tal variable sí afecta la supervivencia del arbolado, por ejemplo, en RodríguezTrejo et al. (2007), para P. hartwegii y en Ryan y Frandsen (1991), para P. ponderosa Laws, en EE. UU. En ambas fuentes se señala que, en los pinares abiertos, los combustibles dominantes son zacates, mientras que en las masas densas domina la hojarasca. Cuando los primeros se queman liberan más calor hacia arriba, afectando parcialmente copas, daño del que muchos pinos se recuperan. Sin embargo, cuando se quema la hojarasca, hay mayor combustión residual, se transmite calor letal a las 
raíces superficiales y esto causa mayor mortalidad, aunque no necesariamente se afecte mucho a las copas.

Pausas, Lamont, Paula, Appezzato-da-Glória y Fidelis (2018) refieren que las especies que cuentan con yemas en el cuello de la raíz son principalmente árboles y que están adaptadas a incendios de regular severidad y, aunque este carácter no les permite extenderse vegetativamente, sí les facilita persistir en el sitio. Un ejemplo de la mayor supervivencia postincendio en especies tolerantes al fuego, respecto a las resistentes, lo refiere Rodríguez-Trejo (2014) para la Sierra de Los Ajos, Sonora, luego de un incendio natural de copas en una masa de Pinus leiophylla var. chibuabuana Shaw. y Q. arizonica Sarg. El fuego mató a todos los pinos y la parte aérea de los encinos, pero estos últimos rebrotaron y 14 años más tarde ya habían recuperado una parte importante de su biomasa aérea. Es conveniente repetir el presente estudio en la actualidad, a 12 años de los incendios forestales.

\section{CONCLUSIONES}

$\mathrm{El}$ incendio forestal fue de regular intensidad y severidad. La mayor Pm en $P$. montezumae se concentra en los individuos con menor diámetro y altura. En tal pino, $A$. xalapensis y Quercus spp., a menores dimensiones (y por ende mayor juvenilidad), y a mayor afectación de la copa en el caso de P. montezumae, se tendrá mayor Pr. Las latifoliadas A. xalapensis, A. acuminata y Quercus spp., mostraron mayor supervivencia que los pinos y también son mejores rebrotadoras, su estrategia de supervivencia ante el fuego es de tolerancia. La de P. montezumae y $P$. teocote es de resistencia al fuego, en tanto que $P$. patula tuvo mortalidad total, por tratarse de individuos de bajo porte y por ser menos resistente al fuego, pues una adaptación relevante al fuego en esta especie es mediante recolonización de áreas incendiadas, gracias a que sus semillas sobreviven en conos serótinos. En sitios donde la probabilidad de mortalidad se relaciona directamente con la densidad del arbolado, el control de la densidad y el manejo de combustibles pueden ayudar a reducirla en caso de incendio. Los resultados con este tipo de modelos son de utilidad para estimar y predecir efectos del fuego, para definir áreas potenciales en las cuales realizar actividades de manejo de combustibles, en la realización de quemas prescritas (a efecto de prevenir mortalidad en el arbolado) y para precisar si en un área afectada por el fuego se requerirá o no de tareas de reforestación y restauración y en qué medida.

\section{REFERENCIAS}

Agee, J. K. (1993). Fire ecology of Pacific northwest forests. Washington, D. C.: Island Press.

Byram, G. M. (1959). Forest fire behavior. En K. P. Davis (Ed.), Forest fire: control and use (pp. 90-123, 554-555). New York: McGraw-Hill.

Centro Nacional de Estudios Municipales [CNEM]. (1987). La enciclopedia de los Municipios de México. Los Municipios de Puebla. México: Secretaría de Gobernación.

Challenger, A. \& Soberón, J. (2008). Los ecosistemas terrestres. En J. Sarukhán K. (ed.), Capital natural de México (pp. 87-108). México: Conabio.

Comisión Nacional para el Conocimiento y Uso de la Biodiversidad [Conabio] (2021). Alnus acuminata. Recuperado de http://www.conabio.gob.mx/conocimiento/info_especies/arbo les/doctos/9-betul1m.pdf

Comisión Nacional Forestal [Conafor] (2009). Reporte semanal de resultados de incendios forestales 2009. Datos acumulados del 01 de enero al 31 de diciembre de 2009. México: Conafor.

Comisión Nacional Forestal [Conafor] (2020). Reporte semanal nacional de incendios forestales 2020. Cierre del 01 de enero al 31 de diciembre de 2020. Recuperado de https://www.gob.mx/cms/uploads/attachment/file/604834/Ci erre_de_la_Temporada_2020.PDF

Díaz-Hernández, D., Rodríguez-Laguna, R., Rodríguez-Trejo, D. A., Acevedo-Sandoval O. A., \& Maycotte-Morales, C. C. (2014). Tolerancia al fuego de Alnus arguta y Arbutus xalapensis en Singuilucan, Hidalgo. Revista Iberoamericana de Ciencias, 1(7), 103112.

Fonseca G., J., de los Santos P., H., Llanderal C., Cibrián T., D., Rodríguez-Trejo, D. A., \& Vargas H., J. J. (2008). Ips e insectos barrenadores en árboles de Pinus montezumae dañados por incendios forestales. Madera y Bosques, 14(1), 69-80. doi: $10.21829 /$ myb.2008.1411220

Fryer, J. L. (2011). Alnus incana. Fire Effects Information System. USDA, Forest Service. Recuperado de https://www.fs.fed.us/database/feis/plants/tree/alninc/all.html 
Fryer, J. L. (2014). Alnus rhombifolia. Fire Effects Information System. USDA, Forest Service. Recuperado de https://www.fs.fed.us /database/feis/plants/tree/alnrho/all.html

García de Miranda, E. (1998). Modificaciones al sistema de clasificación climática de Köppen. México: UNAM. Recuperado de http://www.publicaciones.igg.unam.mx/index.php/ig/catalog/ book $/ 83$

Hardesty, J., Myers, R. L., \& Fulks, W. (2005). Fire, ecosystems and people: a preliminary assessment of fire as a global conservation issue. The George Wright Forum, 22(4), 78-87.

Hartmann, H. T., Kester, D. E., Davies Jr., F. T., \& Geneve, R. L. (2011). Hartmann \& Kester's Plant propagation (8th ed.). Boston: Prentice Hall.

Hernández-Correa, R., Rodríguez-Trejo, D. A., \& Cruz-Reyes, A. (2019). Near zero mortality in juvenile Pinus hartwegii Lindl. after an extensive prescribed burn and comparison with a forest fire. iforest, 12(4), 397-402. doi: 10.3832/ifor2760-012

Hosmer Jr., D. W., Lemeshow, S., \& Sturdivant, R. X. (2013). Applied logistic regression. (3a ed.). Hoboken, New Jersey: John Wiley \& Sons.

Juárez B., J. E., Rodríguez-Trejo, D. A., \& Myers, R. L. (2012). Fire tolerance of trees in oak-pine forest at Chignahuapan, Puebla, Mexico. International Journal of Wildland Fire, 21(7), 873-881. doi: 10.1071/WF11134

Keeley, J. E., \& Pausas, J. G. (2017). Epicormic resprouting in fire-prone ecosystems. Trends in Plant Science, 22(12), 1008-1015. doi: 10.1016/j.tplants.2017.08.010

López M., M. A., Rodríguez-Trejo, D. A., Santiago-Cortés, F., SerenoChávez, V. A., \& Granados Sánchez, D. (2015). Tolerancia al fuego en Quercus magnoliifolia. Revista Árvore, 39(3), 523-533.

Miller, M. (2000). Fire autecology. En J. K. Brown, \& J. Kapler Smith (Eds.), Wildland fire in ecosystems. Effects of fire on flora (pp. 9-34). General Technical Report RMRS-GTR.42, vol. 2. Ogden, UT: USDA Forest Service, Rocky Mountain Research Station.

New, T. R. (2014). Insects, fire and conservation. Cham: Springer.

Pantoja C., V., Rodríguez-Trejo, D. A., Myers, R. L., Hernández A., E., \& González S., M. V. (2018). Modelación de la mortalidad de Pinus oocarpa en áreas de quema prescrita, incendio conducido e incendio forestal, en Chiapas, México. Acta Universitaria, 28(4), $1-$ 9 .

Pausas, J. G. (2015). Evolutionary fire ecology: lessons learned from pines. Trends in Plant Science, 20(5), 318-324. doi: 10.1016/j.tplants.2015.03.001
Pausas, J. G., Lamont, B. B., Paula, S., Appezzato-da-Glória, B., \& Fidelis, A. (2018). Unearthing belowground bud banks in fireprone ecosystems. New Phytologist, 217, 1435-1448. doi: $10.1111 /$ nph.14982

Robles G., C. A., Velázquez M., A., Rodríguez-Trejo, D. A., Reyes H., V. J., \& Etchevers B., J. D. (2016). Probability of mortality by fire damage of young Pinus hartwegii Lind. trees in the Izta-Popo National Park. Revista Chapingo. Serie Ciencias Forestales y del Ambiente, 22(2), 165-178. doi: 10.5154/r.rchscfa.2015.08.034

Rodríguez-Trejo, D. A. (2008). Fire regimes, fire ecology, and fire management in Mexico. Ambio, 37(7/8), 548-556. doi: 10.1579/0044-7447-37.7.548

Rodríguez-Trejo, D. A. (2014). Incendios de vegetación. Su ecología, manejo e historia (Vol. 1). México: C. P. - UACH - Semarnat - PPCIF PNIP - Conafor - Conanp - ANCF - AMPF.

Rodríguez-Trejo, D. A. (2015). Incendios de vegetación. Su ecología, manejo e bistoria (Vol. 2). México: C. P. - UACH - Semarnat - PPCIF PNIP - Conafor - Conanp - ANCF - AMPF.

Rodríguez-Trejo, D. A. \& Fulé, P. Z. (2003). Fire ecology of Mexican pines and a fire management proposal. International Journal of Wildland Fire, 12(1), 23-37. doi: 10.1071/WF02040

Rodríguez-Trejo, D. A., Pausas, J. G., \& Miranda, A. G. (2019). Plant responses to fire in a Mexican arid shrubland. Fire Ecology, 15(11), 1-9. doi: 10.1186/s42408-019-0029-9

Rodríguez-Trejo, D. A., Rodríguez-Aguilar, M., Fernández-Sánchez, F., \& Pyne, S. J. (2001). Educación e incendios forestales. México: Mundi Prensa.

Rodríguez-Trejo, D. A., Castro-Solís, U. B., Zepeda-Bautista, E. M. A., \& Carr, R. J. (2007). First year survival of Pinus hartwegii Lindl. in burned areas in different times. International Journal of Wildland Fire, 16, 54-62. doi: 10.1071/WF05061

Rodríguez-Trejo, D. A., Martínez-Muñoz, P., \& Martínez-Lara, P. (2019). Efectos del fuego en el arbolado de un bosque tropical de pino y en el de una selva baja caducifolia en Villaflores, Chiapas. Ciencia Florestal, 29(3), 1033-1047. doi: 10.5902/1980509833952

Romero R., S., Rojas Z., E. C., \& Rubio L., L. E. (2015). Descripción morfológica de 100 especies de Quercus en México. En S. Romero R., E. C. Rojas Z., \& L. E. Rubio L. (Coords.). Encinos de México. (pp. 129-288). México: UNAM.

Ryan, K. C. (1982a) Evaluating potential tree mortality from prescribed burning. En D. M. Baumgartner (Ed.), Site preparation and fuels management on steep terrain (pp. 167-179). Spokane, WA: Washington State University. 
Ryan K. C. (1982b). Techniques for assessing fire damage to trees. En J. E. Lotan (Ed.), Proceedings of the Symposium: Fire, its field effects (pp. 1-11). 19-21 October 1982, Jackson, WY: Intermountain Fire Council.

Ryan, K. C. (1990). Predicting prescribed fire effects on trees in the interior west. Forestry Canada, Northwest Region Information Report NOR-X-309.

Ryan, K. C., \& Frandsen, W. H. (1991). Basal injury from smoldering fires in mature Pinus ponderosa Laws. International Journal of Wildland Fire, 1, 107-118. doi: 10.1071/WF9910107

Ryan, K. C., \& Reinhardt, E. D. (1988). Predicting post-fire mortality of seven western conifers. Canadian Journal of Forest Research, 18, 1291-1297. doi: 10.1139/x88-199

Statistical Analysis Sytem [SAS ] Institute. (2012). SAS Program® ${ }^{\circledR}$. Cary, North Carolina: SAS Institute.

Scott, A. C., Bowman, D. M. J. S., Bond, S. J., Pyne, S. J., \& Alexander, M. E. (2014). Fire on Earth: An introduction. Chichester: Wiley Blackwell.

Shlisky, A., Waugh, J., González, P., González, M., Manta, M., Santoso, H., Alvarado, E., Nuruddin, A. A., Rodríguez-Trejo, D. A., Swaty, R., Schmidt, D., Kaufmann, M., Myers, R., Alentar, A., Kearns, F., Jonson, D., Smith, J., Zollner, \& D., Fulks, W. (2007). Fire, ecosystems and people: Threats and strategies for global biodiversity conservation. GFI Technical Report 2007-02. Arlington, VA: The Nature Conservancy.

Temiño V., S., Rodríguez-Trejo, D. A., Molina F., D., \& Ryan, K. (2016). Modelling initial mortality of Abies religiosa in a Crown fire in Mexico. Forest Systems, 25(1), 1-12. doi: 10.5424/fs/201625106887

Tinuhua, H., Pausas, J. G., Belcher, C. M., Schwilk, D. W., \& Lamont, B. B. (2012). Fire-adapted traits of Pinus arose in the fiery Cretaceous. New Phytologist, 194, 751-759. doi: 10.1111/j.14698137.2012.04079.x
Vera-Vilchis, V. \& Rodríguez-Trejo, D. A. (2007). Supervivencia e incremento en altura de Pinus hartwegii a dos años de quemas prescritas e incendios experimentales. Agrociencia, 41(2), 219-230.

Whelan, R. J. (1997). The ecology of fire. Cambridge: Cambridge University Press.

Woolley, T., Shaw, D. C., Ganio, L. M., \& Fitzgerald, S. (2011). A review of logistic regression models used to predict post-fire tree mortality of Western North America conifers. International Journal of Wildland Fire, 21(1), 1-35. doi: 10.1071/WF09039

World Wide Fund for Nature \& Boston Consulting Group [WWF \& BCG]. (2020). Fires, forests and the future. A crisis raging out of control? Recuperado de https://wwfeu.awsassets.panda.org/downloads/wwf_forestfires report2020_final.pdf

Zavala Ch., F. (2007). Guía de los encinos de la sierra de Tepotzotlán, México. México: UACH.

Manuscrito recibido el 12 de abril de 2020

Aceptado el 28 de marzo de 2021

Publicado el 24 de diciembre de 2021

Este documento se debe citar como:

Gómez-Mendoza, F. F., \& Rodríguez-Trejo D. A. (2021). Fuego, mortalidad y rebrotación en especies forestales de la Sierra Norte de Puebla. Madera y Bosques, 27(3), e2732148. doi: 10.21829/myb.2021.2732148

Madera y Bosques por Instituto de Ecología, A.C. se distribuye bajo una Licencia Creative Commons Atribución-NoComercialCompartirlgual 4.0 Internacional. 